\title{
LA INTERCULTURALIDAD Y LOS ITINERARIOS CULTURALES EN AMÉRICA LATINA ${ }^{1}$
}

\author{
Joan FELIU FRANCH \\ Universitat Jaume I
}

\begin{abstract}
RESUMEN: El texto plantea cómo la comunicación intercultural en el campo del patrimonio material e inmaterial ha ido ganando peso en las instituciones internacionales, y especialmente en el contexto latinoamericano, bajo el auspicio de la UNESCO.
\end{abstract}

PALABRAS CLAVE: Patrimonio, cultura, hispanoamérica, comunicación, interpretación.

ABSTRACT: The article shows how intercultural communication in the field of tangible and intangible heritage has been gaining weight in international institutions, especially in Latin American countries under the auspices of UNESCO.

KEYWORDS: Heritage, culture, Hispanic American, communication, interpretation.

En las siguientes líneas queremos reflexionar sobre cómo la comunicación intercultural en el campo del patrimonio material e inmaterial ha ido ganando peso en las instituciones internacionales, y especialmente en el contexto latinoamericano, bajo el auspicio de la UNESCO.

Las sociedades latinoamericanas de hoy se han tornado, en los últimos años, cada vez más multiculturales, debido a la inmigración, generalmente interna, y al movimiento de las gentes en general, por lo que son muchas las instituciones que, amparadas por las últimas recomendaciones de la UNESCO, se han ido dando cuenta de la necesidad de proteger la interculturalidad, es decir, el diálogo entre todas las culturas, en un espacio definido y compartido.

La UNESCO, junto con otros determinados organismos internacionales, constituyen los guardianes universales de los monumentos y sitios ${ }^{2}$. A lo largo de

\footnotetext{
${ }^{1}$ Recibido el 1 de julio de 2011. Aceptado en el consejo de 8 de noviembre de 2011.
} 
su historia la UNESCO ha puesto en marcha una valiosa red internacional de convenciones, cartas y recomendaciones. Este conjunto de textos plasma las reglas que deben permitir gestionar correctamente la conservación del patrimonio a escala mundial y nacional, así como su eficaz protección contra los daños del tiempo y de los hombres.

Así mismo, ha preconizado o fomentado la constitución de organizaciones internacionales especializadas, no sólo intergubernamentales sino también gubernamentales como es el Consejo Internacional de Museos (ICOM), creado en 1946, y más tarde el Instituto Internacional para la Conservación (IIC), en 1950, así como el Centro Internacional de Estudios y Restauración de Bienes Culturales, en 1959 y, por fin, el Consejo Internacional de Monumentos y Sitios (ICOMOS) que nació al mismo tiempo que la Carta de Venecia, en 1964.

Es obvio que el patrimonio cultural y natural, tangible e intangible, forma parte de los bienes inestimables e irremplazables no sólo de cada nación sino de toda la humanidad $^{3}$. La pérdida de uno de estos bienes eminentemente valiosos, como resultado de su degradación o desaparición, constituye un empobrecimiento del patrimonio de todos los pueblos del mundo. Se puede considerar que algunos elementos de este patrimonio, por sus notables calidades, tienen un valor universal excepcional y merecen, por ende, una protección especial contra los peligros crecientes que los amenazan ${ }^{4}$.

Con el fin de mediar esta situación crítica y de garantizar lo mejor posible la identificación, la protección, la conservación y la revalorización adecuadas de este patrimonio mundial irremplazable, los Estados Miembros de la UNESCO aprobaron en 1972 la Convención para la Protección del Patrimonio Mundial Cultural y Natural, denominada comúnmente la Convención. Ésta completaba los programas nacionales de conservación del patrimonio y preveía el establecimiento

${ }^{2}$ SILVA, R. (2002). «El papel de ICOMOS con relación a la UNESCO, los Estados Parte, los Comités Nacionales y Comités Científicos Internacionales», Simposio XIII Asamblea General de ICOMOS, Madrid, [recurso electrónico] http://www.icomos-ciic.org. [Consultado 01/06/2011].

${ }^{3}$ CPPM (1999) Directrices prácticas sobre la aplicación de la Convención para la Protección del Patrimonio Mundial del Comité Intergubernamental de Protección del Patrimonio Mundial Cultural y Natural en su revisión aprobada por el Comité del Patrimonio Mundial en su 22 reunión de diciembre de 1998, UNESCO, París, [recurso electrónico]. http://www.unesco.org/whc. [Consultado 01/06/2011].

${ }^{4}$ Dadulatli, A. (2002). «Conservación del Patrimonio Mundial. De la filosofía a la práctica: la Convención del Patrimonio Mundial como referencia para los bienes culturales», Simposio XIII Asamblea General de ICOMOS, Madrid, [recurso electrónico].. http://www.icomos-ciic.org. [Consultado 01/06/2011]. 
de un Comité del Patrimonio Mundial, así como la constitución de un Fondo del Patrimonio Mundial. Tanto el Fondo como el Comité fueron creados en 1976 con la premisa de aprovechar otros instrumentos internacionales como la Convención de Ginebra de 1949, la Convención de la Haya de 1954, la Convención de la UNESCO de 1970, la Convención de Ramsar, o la CITES.

Dentro de la Lista de Patrimonio Mundial, y terminando con la ignorancia que se había ejercido históricamente sobre el valor los fenómenos de intercambio cultural, los tipos de bienes se ampliaron recientemente con la consideración patrimonial de los llamados itinerarios culturales (en inglés cultural routes y en francés itinéraire culturel).

En el propio ICOMOS existe una organización específica dedicada al estudio de los itinerarios culturales, el CIIC (Comité Científico Internacional de Itinerarios Culturales), que tiene como objetivos identificar, describir, analizar, conservar y promocionar itinerarios culturales auténticos; dicha promoción va encaminada a las vertientes cultural, económica y social, así como a fomentar el interés y la solidaridad en torno al patrimonio compartido por distintos pueblos y comunidades culturales $^{5}$.

En realidad, el hecho de que la intercomunicación cultural pudiera ser protegida por la UNESCO es consecuencia de una propia acción de esta organización: la inscripción del Camino de Santiago como Patrimonio Mundial en 1993. Con motivo del interés despertado por dicha inscripción, se celebró en Madrid en noviembre de 1994 un congreso con el título de Los Itinerarios como parte de nuestro Patrimonio Cultural encaminado a precisar este término ${ }^{6}$.

Sin duda influyó también el conocido como Documento de Nara sobre la Autenticidad, un texto del mismo 1994, concebido en el espíritu de la Carta de Venecia de 1964, que intentaba dar respuesta desde la UNESCO al alcance creciente de las preocupaciones e intereses del patrimonio cultural en el mundo contemporáneo. El Documento de Nara era en realidad el resultado redactado por los ponentes generales Raymond Lemaire y Herb Stovel, de la Conferencia de Nara sobre la Autenticidad en Relación con la Convención sobre el Patrimonio Mundial,

5 CIIC (1999-2003). Seminario Internacional del CIIC, Guanajuato, [recurso electrónico]. http://www.icomos-ciic.org/INDEX_esp.htm. CIIC, Meeting of experts on cultural routes, Madrid, http://www.icomos-ciic.org/INDEX_esp.htm. [Consultado 01/06/2011].

${ }^{6}$ SuAREZ-InClán DuCASSI M. R., (2003) «Los itinerarios culturales», Ponencia presentada el 15 de diciembre del 2003 en el Encuentro Internacional sobre: La Representatividad en la Lista del Patrimonio Mundial. El Patrimonio Cultural y Natural de Iberoamérica, Estados Unidos y Canadá, Querétaro. 
celebrada en Nara, Japón, del 1 al 6 de noviembre de 1994, a instancias de la Agencia de Asuntos Culturales (Gobierno de Japón) y de la Prefectura de Nara, y en cooperación con la UNESCO, el ICCROM y el ICOMOS.

En una América cada vez más sujeta a las fuerzas de la globalización y la homogeneización, y en la cual la búsqueda de la identidad cultural se persigue en ocasiones a través de nacionalismos agresivos o de la supresión de las culturas minoritarias, la UNESCO comenzaba a preocuparse por la conexión entre la diversidad de las culturas, y como esta podía incluso ser motivo de controversia para certificar la autenticidad que se le demandaba a cualquier bien que quisiera ser considerado Patrimonio Mundial.

Como venía a decir el texto de Nara, si la comunicación entre la diversidad de culturas y de patrimonios en nuestro mundo es una fuente irreemplazable de riqueza, tanto espiritual como intelectual, para toda la humanidad, es obvio que la protección y favorecimiento de esta comunicación en la diversidad cultural y patrimonial en nuestro mundo debería promoverse de manera activa como un aspecto esencial del desarrollo humano, y formar parte de los objetivos de la UNESCO en general y de la Convención del Patrimonio Mundial en particular.

Cabría, no obstante, tener en cuenta que la diversidad del patrimonio cultural se interrelaciona, se comunica, existe en el tiempo y en el espacio, de forma que todas las culturas y las sociedades están enraizadas en formas y medios particulares de expresión tangibles e intangibles que constituyen su patrimonio y que deberían ser respetados.

De hecho, todos los juicios sobre valores que se atribuyan a los bienes culturales, así como la credibilidad de las fuentes de información relacionadas, pueden variar de una cultura a otra, e incluso dentro de la misma cultura. Por lo tanto, no es posible basar juicios sobre el valor y la autenticidad con criterios inamovibles.

Al contrario, el respeto debido a todas las culturas requiere que los bienes del patrimonio deban juzgarse y tomarse en consideración dentro de los contextos culturales a los que pertenecen, y la continuación y ampliación de este diálogo entre las diversas regiones y culturas del mundo constituye un prerrequisito para aumentar el valor práctico de la toma en consideración de la autenticidad para la preservación del patrimonio común de la humanidad.

La concienciación cada vez mayor del público en lo que respecta a esta dimensión fundamental del patrimonio es una necesidad absoluta hoy en día para la UNESCO, sin embargo, la comunicación cultural, representada internacionalmente 
en el campo de la cultura tangible e intangible por los itinerarios culturales, no se puede inventar.

No hay una comunicación cultural protegible desde un punto de vista patrimonial en el establecimiento artificial de conjuntos asociativos de bienes culturales que poseen rasgos comunes. No estamos hablando, por tanto, de la ruta de la lengua española que vincula por meros motivos de oportunidad política a tres o cuatro puntos geográficos próximos entre sí, o de las rutas de escritores o artistas entre las poblaciones en las que cada uno de ellos vivieron aunque no se hubieran conocido nunca. Un itinerario cultural no es una mera asociación de ideas o elementos.

Su definición no llegaría hasta la segunda reunión de expertos, celebrada también en Madrid en Mayo de 2003. La directiva internacional del CIIC fue informada de que en la Sexta Reunión Extraordinaria del Comité de Patrimonio Mundial, celebrada los días 17 y 22 de marzo de 2003, a raíz de las intervenciones hechas por la delegación oficial de México y de otros países, se solicitó de ICOMOS la preparación de una propuesta dirigida a considerar la posible inclusión de los itinerarios culturales como un nuevo concepto específico entre los diferentes bienes culturales contemplados en las Directrices de la Convención del Patrimonio Mundial, y se redactó la definición en los siguientes términos:

Debe entenderse por itinerario cultural toda vía de comunicación terrestre, acuática o de otro tipo, físicamente determinada y caracterizada por poseer su propia y específica dinámica y funcionalidad histórica que reúna las siguientes características: a) ser resultado y reflejo de movimientos interactivos de personas, así como de intercambios multidimensionales, continuos y recíprocos de bienes, ideas, conocimientos y valores dentro de un país o entre varios países y regiones, a lo largo de considerables períodos de tiempo; b) haber generado una fecundación múltiple y recíproca de las culturas en el espacio y en el tiempo que se manifiesta tanto en su patrimonio tangible como intangible ${ }^{7}$.

Las dos siguientes precisiones abonan el concepto y resaltan el significado y funcionalidad de los itinerarios culturales:

Teniendo en cuenta la riqueza y variedad tanto de las interrelaciones como de los bienes culturales que pueden existir dentro de un itinerario cultural

${ }^{7}$ SuÁREZ-InClán DuCASSI, M. R. (2001) «Los itinerarios culturales», Congreso Internacional del CIIC de ICOMOS, Pamplona, [recurso electrónico]. http://www.icomos-ciic.org. [Consultado 01/06/2011]. 
(monumentos, restos arqueológicos, arquitectura vernácula, patrimonio industrial y tecnológico, obras públicas, paisajes culturales, medios de transporte y otros exponentes de la aplicación de conocimientos específicos y habilidades técnicas), los itinerarios culturales constituyen un instrumento idóneo para ilustrar el hecho de que la realidad cultural es una evidencia polifacética que requiere una aproximación multidisciplinar. Así mismo renuevan las hipótesis científicas y permiten acrecentar los conocimientos técnicos, artísticos y culturales.

Resultado de encuentros pacíficos o controvertidos, actualmente los itinerarios culturales entrañan una gran variedad de dimensiones compartidas que trascienden su primitiva función ofreciendo un cuadro privilegiado para una lectura plural de la historia, así como una cultura de paz y entendimiento mutuo basados en la cooperación entre los pueblos.

Así pues, los itinerarios culturales representan un cambio cualitativo de la noción de conservación del patrimonio que permite una vinculación cultural entre pueblos, ciudades, regiones y continentes, porque ofrecen la oportunidad vincular el territorio con un patrimonio intangible importante para la vida tradicional de las comunidades implicadas en su trayecto.

Los itinerarios culturales son los que tendieron los puentes de la comunicación, el intercambio y el entendimiento entre distintos pueblos. A través de ellos, nos mezclamos, y dimos lugar a una diversidad cultural con identidades compartidas y diferentes matices y, al mismo tiempo, con personalidad local propia en el patrimonio construido e intangible. Todos y cada uno de los pueblos han aportado conocimiento y cultura que, merced al efecto multiplicador de los intercambios, se han ido introduciendo entre la humanidad, adoptando formas y características propias en cada caso. El análisis e interpretación de esos cruces permiten afirmar nuestros rasgos peculiares y característicos y, simultáneamente, nos proporcionan otra imagen más integral de nosotros mismos.

Para la UNESCO, en un contexto universal, no es válida la idea de seres y países individualistas encerrados en una dinámica meramente interna. Especialmente ante los aspectos negativos de la globalización actual, interesa reconocer que formamos parte de grupos culturales unidos por factores comunes de cohesión entre nuestras respectivas identidades, así como por intereses compartidos. Los itinerarios culturales nos brindan la oportunidad de revalorizar nuestra propia identidad, y también nuestros principios como individuos y como grupo cultural.

Sin embargo, aún quedan cuestiones por resolver, más allá de la difícil clasificación de estos itinerarios culturales. Hace ya unos diez años que se 
comenzaron a abordar las problemáticas que conlleva el itinerario cultural, pero aún así continúan ciertas indefiniciones.

Si tuviéramos que destacar avances significativos, no nos quedaría más remedio que alejarnos en el tiempo hasta los momentos en que estos temas se estaban configurando. Así, la $31^{\mathrm{a}}$ conferencia general de la UNESCO, Cultural Diversity and Heritage Preservation at the Heart of Mutual Understanding (La diversidad cultural y la preservación del patrimonio en el corazón de la comprensión mutua), celebrada en París, del 15 de octubre al 3 de noviembre del 2001, con la asistencia de doscientos treinta ministros y viceministros de cultura, aprobó una Declaración Universal sobre la Diversidad Cultural, que hizo hincapié en valores como el respeto, el intercambio, la tolerancia, el diálogo y la cooperación entre diferentes pueblos y culturas. Esta es la base fundamental del posterior desarrollo de los itinerarios culturales, pero no el único acuerdo.

Casi a la vez, el II Campus Euroamericano de Cooperación Cultural, celebrado en Cartagena de Indias, del 10 al 14 de diciembre de 2001, organizado por el Ministerio de Cultura de Colombia en colaboración con Interarts, la Organización de Estados Iberoamericanos (OEI) y el Convenio Andrés Bello, atrajo a unos 175 participantes del sector público, del sector privado y del mundo universitario que acudieron a la conferencia para discutir de cuestiones relativas a las políticas culturales de Europa y de América. Una de las principales temáticas de la conferencia residió en la interculturalidad, es decir el proceso de mejorar, aumentar y mantener el diálogo entre distintas culturas dentro de las naciones y entre ellas. Los participantes hablaron de consolidar el diálogo intercultural y la cooperación con el fin de asegurar que todas las culturas se respeten y se compartan en un planeta cada vez más mundializado.

El Primer seminario hemisférico de expertos en diversidad cultural para la organización de los estados americanos, Prosperidad, desarrollo y paz: diversidad cultural en las Américas, celebrado en Vancouver, del 17 al 19 de marzo de 2002, como iniciativa de seguimiento a la Cumbre de las Américas de 2001, reunió expertos de veintinueve países que comentaron las maneras de impulsar políticas culturales nacionales en un entorno mundializado y examinaron las políticas culturales como factor clave de las estrategias de desarrollo. El seminario tenía por objeto ofrecer a los expertos la oportunidad de intercambiar informaciones, sus opiniones y preocupaciones relativas a cuestiones de gran relevancia para las políticas culturales del hemisferio, y someter recomendaciones al Grupo de Trabajo de la OEA sobre la Cultura, para su consideración durante la reunión de los Ministros de Cultura en Cartagena, en julio de 2002. Los expertos coincidieron en afirmar que los estados desempeñan un papel fundamental en el desarrollo de 
industrias culturales que florecen y que tienen el derecho de producir instrumentos para proteger su diversidad y su patrimonio cultural. Hicieron un llamamiento para una mayor cooperación en cuestiones relacionadas con la cultura y recomendaron la creación de un observatorio de políticas culturales, de una base de datos sobre patrimonio cultural, así como de un mapa cultural americano.

Por último, para no ser redundante, nos gustaría mencionar la Panam, Diálogo sobre industrias culturales entre las civilizaciones de las Américas, celebrada en Montreal, del 22 al 24 de abril de 2002, que fue organizada por GRICIS, grupo de investigación interdisciplinaria sobre comunicación, información y sociedad, con sede en Montreal. Este grupo se dedica a estudiar el papel que desempeñan los programas de cultura y de comunicación y el impacto de las correspondientes políticas en la sociedad. La conferencia tenía por objeto propiciar el diálogo y la cooperación entre las industrias dedicadas a la cultura y a la comunicación y los académicos, investigadores y representantes de los sectores público y privado, y de desarrolló en torno a cuatro grandes temas: el papel del estado con respecto a la diversidad cultural; las lenguas usadas en los intercambios culturales; el lugar que ocupa la cultura en la economía; y la participación de las primeras naciones en los intercambios culturales.

Finalmente se aprobó la Convención, adoptada por la 33 Conferencia General de la UNESCO en Octubre 2005, aunque no entró en vigor hasta el 18 de Marzo 2007, que bajo el título: Convención sobre la protección y la promoción de la diversidad de las expresiones culturales, aspira a crear un entorno propicio en el que la diversidad de las expresiones culturales pueda afirmarse y renovarse en interés de todas las sociedades. La idea es reforzar los cinco eslabones inseparables de una misma cadena: la creación, la producción, la distribución / diseminación, el acceso y el disfrute de las expresiones contenidas en las actividades, los bienes y los servicios culturales

En todo caso, la consecuencia práctica de esta manera de entender el patrimonio a principios de este siglo fue el impulso dado a un gran itinerario cultural panamericano.

Qué duda cabe de que el estado del patrimonio cultural de muchos itinerarios culturales americanos es preocupante. Su integridad cultural se halla destruida, su patrimonio común está fragmentado en sistemas nacionales cerrados y, en la mayoría de los casos, es poco conocido en el mundo. No existen políticas coordinadas para la protección y promoción del patrimonio. Las crisis económicas, la legislación obsoleta de los países en transición, los conflictos, tanto militares como étnicos, y las catástrofes naturales no solo afectan negativamente sino que 
ponen en serio peligro al patrimonio cultural que resulta muy vulnerable. La identificación, el estudio y la promoción de los itinerarios culturales debe ayudar a poner fin a este estado de cosas, lanzando y divulgando la idea de este nuevo papel que corresponde al patrimonio cultural y definir, por vez primera, la macroestructura del patrimonio dentro de los itinerarios culturales regionales e internacionales. El patrimonio debe ser identificado como un sistema, una comunidad unida que cuenta con sus propias infraestructuras, redes de células, zonas y centros, y no solamente como una mera suma de sistemas nacionales aislados.

Solventar estos problemas es lo que pretendía, por ejemplo, el proyecto del Qhapaq Ñan, el itinerario cultural más extenso y ambicioso de Sudamérica.

El origen del interés por recuperar el Qhapaq Ñan hay que buscarlo en las investigaciones que Jhon Hyslop realizó entre 1978 y 1981, materializadas en el libro The Inca Roat System publicado en 1984 por Academic Press, y traducido parcialmente al castellano en 1992. Estos trabajos completaban los primeros acercamientos al camino Inca que ya habían realizado Alberto Regal (Los caminos del inca, 1936); Víctor Von Hagen (Los caminos del Sol, 1958); o León Strube Erdmann (con sus clases en la Universidad de Córdoba, Argentina, en 1963), y despertaron el interés sobretodo en Chile, Argentina, Bolivia, Perú y Ecuador.

No se trata de caminos hechos al andar, ni caminos locales entre poblaciones vecinas, sino el resultado de un ambicioso proyecto vial para mejorar la comunicación comercial y política de un vastísimo territorio. Gracias a estos caminos, productos, gentes e ideas circularon a lo largo de cientos de kilómetros uniformando el territorio del Tawantisuyu inca.

En la actualidad su importancia cobra vigencia al convertirse en un elemento ordenador del irregular espacio internacional andino. Hay que tener en cuenta que el Tahuantisuyu fue probablemente el más grande y antiguo imperio desarrollado en el continente americano. Tuvo como sede a la ciudad de Cuzco y data del año 1200 d.C. La palabra Tahuantinsuyu proviene de un nombre compuesto por dos vocablos quechuas: Tawa, que significa cuatro, y Suyo, que quiere decir Estado.

El área territorial del imperio fue vastísima. Ocupó una superficie de más de 3.000 .000 de $\mathrm{Km}^{2}$ que incluía casi 5,000 km de costa sobre el Océano Pacífico, lo que representa hoy poco menos del doble de la costa del territorio peruano.

El mismo nombre, Tahuantinsuyu, nos indica la división del territorio, basada en relaciones de dualidad, tripartición y cuarta-partición, característicos de la mentalidad inca. 
Los cuatro suyos o naciones tenían como centro geográfico y político a Cuzco. Al noroeste se ubicaba el Chinchaysuyu, que iba hasta el Río Ancashmayo en Pasto, Colombia; al noreste el Antisuyu, en los valles subtropicales, ocupando parte de la selva baja amazónica; al sudoeste el Contisuyu ocupaba parte de la costa peruana hasta el Río Maule en Chile, y al sudeste el Collasuyu, que ocupó gran parte del actual territorio boliviano, llegando hasta Tucumán, en el Norte de Argentina.

Con la llegada de la colonia estos caminos fueron sustituidos por los de herradura allá donde se pudo, y muchas zonas fueron olvidadas. Esto hace que en algunos lugares el abandono ha mantenido vivos muchos valores nativos que ahora pueden ser conocidos. Pero no es menos cierto que en otros, los tiempos de la colonia se mezclaron con los incas, dando al Qhapaq Ñan un valor añadido.

Las campañas desarrolladas bajo la coordinación de Guadalupe Martínez Martínez, han ido en esta dirección. Desde la ciudad de Cuzco hasta el Machu Picchu, ambas consideradas como patrimonio de la Humanidad, se extiende el valle sagrado de los Incas, con poblaciones unidas por la red de caminos que estos realizaron y que se integran, como parte fundamental, en el proyecto Qhapaq Ñan que tiene dos subprogramas, uno estrictamente ligado a la región de Cuzco y otro que cubre el resto del país. Todo ello se integra en el espacio natural de la cordillera de los Andes, formando un conjunto que representa el bien de interés cultural más importante del Perú, necesitado de actuaciones destinadas a su preservación y puesta en valor.

El 4 de diciembre del 2010, los presidentes de Argentina, Bolivia, Chile, Colombia, Ecuador y Perú firmaron en Mar del Plata una Declaración conjunta que ratifica el compromiso de preservar de forma colectiva ese patrimonio común, y presentar conjuntamente una candidatura única de inscripción en la Lista de Patrimonio Mundial de la Unesco.

De esta manera se alcanzaría el objetivo específico de ayudar a alcanzar una serie de estrategias por medio de modelos que incidan en un mejor desarrollo del patrimonio a través de la sistematización turística integrada, armonizando la conservación y la dinamización socioeconómica y cultural de un área declarada Patrimonio de la Humanidad, y la posterior exportación de la experiencia a otras zonas.

Hay que abordar de forma global la planificación del proceso de puesta en valor, la identificación y documentación del patrimonio, la capacitación y creación de estructuras de gestión y participación, y la gestión sostenible, en la línea marcada por la Red Iberoamericana para la Revitalización de los Centros 
Históricos, lo que completaría el conocimiento de este importante itinerario cultural.

Las innovaciones y aportaciones de otras culturas, así como los cruces e intercambios producidos a través de este camino, también han dado lugar en el conjunto de América Latina a un inmenso y rico sincretismo, tanto material como espiritual, y a las más variadas manifestaciones de una cultura mestiza con propia personalidad. Los itinerarios culturales fueron una de las vías generadoras de dichos cambios, y a lo largo de su recorrido pueden apreciarse los múltiples testimonios patrimoniales, físicos e intangibles, que atestiguan su existencia y su función de trasvase intercultural recíproco. De hecho, teniendo en cuenta esta realidad, el CIIC reconoce la importancia de "El Camino Real Intercontinental" que constituye un itinerario cultural de gran coherencia histórica y destacada riqueza patrimonial, entre Europa, América y también Filipinas.

Los itinerarios culturales pueden conducirnos a conocer, comprender y vivir la experiencia humana en muy distintos aspectos con una dimensión más reflexiva, más vasta y profunda, en definitiva más enriquecedora. Esto supone igualmente que los itinerarios culturales pueden servir para atraer al público en general hacia una mejor comprensión de una historia y un patrimonio comunes que reflejan el resultado de la fructífera colaboración entre pueblos diversos. De esta forma, el desarrollo turístico puede avanzar a través de la promoción de los itinerarios culturales. Ello significa también que, merced a éstos, pueden establecerse rutas de desarrollo sostenible que se beneficien del turismo cultural, lo cual puede contribuir a paliar numerosos problemas económicos, de abandono y de pobreza de muchas comunidades.

No obstante, de las diferentes reuniones que se han ido sucediendo en estos últimos años extraemos diversas cuestiones que están todavía por resolver, y que nacen del planteamiento americano de la cuestión:

No está claro como se define el vínculo entre la diversidad cultural y los derechos humanos que a todas luces es necesario para preservar el diálogo entre culturas.

No existen políticas concretas tendentes a armonizar la diversidad y la comunicación cultural y la solidaridad internacional, y es obvio que ambos conceptos deben caminar unidos en más ocasiones de las que desearíamos.

Reconocemos que la comunicación cultural histórica a nivel internacional influye en la formulación de las políticas nacionales (como ocurre entre España e Hispanoamérica), pero no se ha definido todavía cómo pueden los estados 
colaborar con la UNESCO y entre ellos en el desarrollo de estrategias para la promoción de los principios de diversidad cultural enunciados en la Declaración del Patrimonio Mundial de una forma paritaria, ni si eso es conveniente. Es como fomentar la diversidad lingüística sin dejar de respetar el idioma vernáculo.

No está definido cual es el papel de la UNESCO a la hora de ayudar a los gobiernos nacionales a mejorar las relaciones interculturales en sus respectivos países. ¿Cómo pueden los estados desarrollar e implantar políticas culturales nacionales para impulsar y preservar la diversidad cultural? ¿Cómo pueden las complejidades y diversas interpretaciones de la diversidad cultural integrarse en un instrumento internacional con fuerza legal?

Tampoco existen estudios importantes de cómo pueden los gobiernos generar oportunidades para el diálogo intercultural que tengan en cuenta a las minorías.

La educación y la cultura necesitan mayor número de programas de acción, ya que la potenciación de la comunicación cultural debe plantearse desde la base de la pirámide hacia arriba, y no lo contrario.

No se pueden pasar por alto la importancia y la necesidad de las redes ni la interacción que existe entre ellas. A cada red le corresponde crear vínculos con otras, ya sean de su misma vocación o no, de manera a intercambiar ideas y experiencias. Esto permitirá asegurar un clima de diálogo permanente con la plena participación de actores de todos los niveles de la sociedad.

En cuanto al impacto de la globalización sobre la cultura y las políticas culturales, existe un consenso respecto a la necesidad de hacer frente a los desafíos que se presentan ante la cultura y determinar la manera en que las políticas culturales pueden ayudar a lograrlo, pero no se han definido en programas concretos.

No obstante, y en definitiva, lo cierto es que la interculturalidad y las relaciones interculturales parecen revestir cada vez mayor importancia entre los criterios por los que se rigen las sociedades con una multiplicidad de etnias, de culturas y de lenguas, a la hora de formular sus políticas y confeccionar sus programas; y que en los últimos diez años la UNESCO ha iniciado un camino, del que todavía faltan definir bastantes márgenes, pero que se aleja ya de la visión del patrimonio como un listado de monumentos, y atiende a la comunicación de la diversidad cultural como el principal legado de la humanidad, un diálogo entre culturas esencial para asegurar una interacción armoniosa entre nuestras sociedades, que son cada vez más diversas, importante para la cohesión social, factor de desarrollo y fuente de 
creatividad. Sólo la UNESCO puede desarrollar instrumentos internacionales con fuerza legal para preservar y fomentar la comunicación y la diversidad cultural.

Dejemos que todas las culturas se expresen, y para ello, la UNESCO debe impulsar la interculturalidad, es decir la necesidad de diálogo, de intercambio y de una interacción armoniosa dentro de las naciones y entre ellas; debe propiciar que los estados formen consorcios y cooperen entre ellos en sus programas relativos a la diversidad cultural; debe promocionar la creación de observatorios sobre diversidad cultural e invitarlos a compartir sus experiencias y a obrar como redes vinculadas entre sí; y debe dejar bien claro en toda su política patrimonial que la democratización de la cultura se percibe como un proceso que ha de empezar en la base de la pirámide y que se tiene que caracterizar por el respeto hacia la diversidad cultural, haciendo especial hincapié en el respeto hacia la comunicación cultural histórica, actual y futura. 